\title{
DEMONSTRAÇÃO E PRÁTICA MENTAL \\ NA AQUISIC̣ÃO DE HABILIDADES MOTORAS
}

\author{
DEMONSTRATION AND MENTAL PRACTICE \\ IN THE ACQUISITION OF THE MOTOR SKILLS
}

\author{
AUTORES \\ Fabiano de Souza Fonseca' \\ Mariana Bassalo Siqueira ${ }^{1}$ \\ Alessandro Teodoro Bruzi ${ }^{1}$ \\ João Vitor Fialho ${ }^{1}$ \\ Herbert Ugrinowitsch ${ }^{1}$ \\ Rodolfo Novellino Benda ${ }^{1}$ \\ 'Grupo de Estudos em Desenvolvimento \\ e Aprendizagem Motora - Escola \\ de Educação Física - UFMG \\ DEMONSTRAÇÃO E PRÁTICA \\ MENTAL NA AQUISIC̣ÃO \\ DE HABILIDADES MOTORAS \\ 4(2): 61-66

\section{PALAVRAS-CHAVE} \\ aprendizagem motora; \\ demonstração; prática mental.
}

\section{KEYWORDS}

motor learning; demonstration; mental practice.

\section{RESUMO}

A demonstração e prática mental são variáveis capazes de realçar a aprendizagem de habilidades motoras. 0 propósito deste ensaio foi apresentar conhecimentos empíricos produzidos nos estudos de demonstração e prática mental, assim como uma possível relação dessas variáveis no processo de aquisição de habilidades motoras. Os resultados dos estudos indicam que essas variáveis atuam similarmente no processo de representação mental da habilidade a ser aprendida. Assim, talvez a associação entre demonstração e prática mental possa representar uma estratégia interessante na aquisição de habilidades motoras.

\section{ABSTRACT}

Demonstration and mental practice are variables capable to enhance the learning of motor skills. The purpose of this study was to show the empirical knowledge produced on demonstration and mental practice studies, as well as, a possible relation between these variables effects in the acquisition of motor skills. The results of studies suggest that these variables act similarly in the process of mental representation of the skill, that will be learned. Thus, perhaps the association of demonstration and mental practice can provide an interesting strategy in the acquisition of motor skills. 


\section{INTRODUĈ̣̃O}

Um dos objetivos das pesquisas realizadas na área de Aprendizagem Motora é investigar os fatores que afetam a aquisição de habilidades motoras. Conhecimento de resultados, observação e demonstração, tipo de instrução, condições de prática, transferência de aprendizagem, imagem e imaginação, entre outros, podem ser citados como tópicos de vários estudos sobre essa temática ${ }^{43}$.

Essas variáveis são testadas isoladamente para verificar o seu papel na aprendizagem de habilidades motoras, ou ainda em associação, já que podem atuar de forma complementar. A demonstração e instrução verbal, prática física e prática mental são exemplos de variáveis que atuam de maneira complementar e, consequentemente, proporcionam maiores benefícios à aquisição de habilidades motoras ${ }^{19,21,46}$.

Como a possibilidade de combinar essas variáveis é grande, ainda há um vasto campo para ser atacado, sendo um exemplo, a interação entre demonstração e prática mental. Este ensaio tem o objetivo de fazer uma revisão dos estudos sobre essas variáveis no campo da aprendizagem motora, tanto dos que manipularam somente uma delas como os que as combinaram.

\section{DESENVOLVIMENTO}

\section{Demonstração}

No processo de aquisição de habilidades motoras, as diferentes formas de fornecer informação anterior à prática podem influenciá-lo. A demonstração e a instrução verbal são as formas mais comuns de fornecimento de informações prévias.
Particularmente, a demonstração tem sido reconhecida como uma importante fonte de informação no processo de aprendizagem de habilidades motoras ${ }^{39}$. A demonstração consiste em fornecer uma imagem representativa da tarefa a ser executada, na qual o meio mais freqüente é a observação de um modelo ${ }^{41,50}$.

A demonstração é definida como o procedimento de fornecer informação sobre a natureza da habilidade a ser desempenhada, que pode ser uma fonte de informação sobre "como fazer"44. Outros autores ainda se referem à demonstração como informação relacionada ao padrão espacial e temporal pelo qual se alcança a meta da tarefa em questão ${ }^{15,43}$.

Devido às suas características, a demonstração é fornecida ao aprendiz anteriormente a execução de uma habilidade motora. Contudo, também pode ser fornecida após a execução, como fonte de informação que serve como referência para correção dos erros de tentativas futuras ${ }^{47,52}$.

A maioria das pesquisas sobre demonstração se fundamenta na teoria da Aprendizagem Social de Bandura ${ }^{9}$, a qual postula que a aprendizagem de um determinado comportamento pode ocorrer pela observação do comportamento e suas conseqüências no ambiente ${ }^{12}$. As informações observadas, como as características espaciais e temporais do padrão de movimento permitem a formulação de uma representação mental da ação a ser realizada ${ }^{9,23,42}$. A representação mental formulada possui duas funções básicas: a primeira relacionada à produção de um modelo interno da ação a ser executada ${ }^{11,12,13,14}$; e a segunda relacionada à formação de um padrão de referência para comparação com o feedback da performance na detecção e correção do erro ${ }^{2,11,12,13,14}$. O modelo interno ou conjunto de regras subjacentes ao comportamento é gerado por quatro processos cognitivos $^{9}$. Os dois primeiros processos, atenção seletiva e retenção, permitem a formulação do plano de ação que conduz ações futuras. 0 processo de atenção seletiva se responsabiliza em determinar o que está sendo seletivamente observado e que permite extrair as informações mais relevantes da ação modelada. 0 processo de retenção se responsabiliza em formular a representação mental da ação com base nas informações extraídas após o processo de atenção seletiva. Algumas atividades mentais, como codificação simbólica das informações selecionadas da ação e ensaio cognitivo, ou seja, prática mental da representação formulada, têm sido eficientes no processo de retenção $0^{7,8,20,28}$. 0 terceiro processo acontece para a produção do movimento, ou seja, a prática da habilidade motora. A representação mental, formulada anteriormente pelos processos de atenção seletiva e retenção, é a referência de informações que serão enviadas aos músculos responsáveis pelo movimento. Além disso, a produção do movimento também será envolvida em um processo de comparação entre a representação interna com o feedback da ação, para posterior correção que seja necessária, o que mostra a importância das tentativas de prática para oportunizar a detecção e correção de erros no processo de produção do movimento ${ }^{9}$. 0 quarto processo é o motivacional, constituído por fatores intrínsecos e ex-trínsecos que incentivam a aprendizagem de habilidades motoras pela observação. Estes fatores têm efeito sobre os processos de atenção seletiva 
e de retenção, e também afetam os observadores motivando-os a executar o movimento aprendido.

A formação do modelo interno acontece de formas diferentes relacionadas à maneira de fornecer informação, que pode ser pela demonstração ou pela instrução verbal. Algumas investigações tiveram o objetivo de comparar as formas de fornecer informação previamente à prática ${ }^{16}$, enquanto outras tiveram o objetivo de identificar "o que" é aprendido".

Com relação ao tipo de informação prévia, no estudo de Dartido ${ }^{16}$ comparou-se a utilização da demonstração e instrução verbal em uma tarefa de subir a escada de BACHMAN. Participaram deste estudo sujeitos com idade entre $11 \mathrm{e}$ 12 anos e os resultados mostraram que o grupo demonstração obteve melhor aprendizagem que o grupo instrução verbal. Resultados semelhantes foram encontrados em outros estudos $^{5,17,33,43}$.

Com o objetivo de verificar "o que" é aprendido, comparou-se os efeitos da demonstração, instrução verbal e a falta de informação em adultos do sexo masculino, em uma tarefa de arremessar um dardo de salão a um alvo circular posicionado paralelamente ao solo ${ }^{4}$. Os efeitos foram avaliados tanto no padrão de movimento, por meio de análise cinemática, como no escore obtido no alvo. Os resultados da análise cinemática revelaram que o grupo demonstração se aproximou mais ao padrão de movimento efetuado pelo modelo que os grupos instrução verbal e controle, ao contrário dos resultados da comparação dos escores, no qual não houve diferença intergrupos. Os resultados sugerem que a demonstração influencia a aquisição da coordenação do movimento por canalizar a busca visual do observador pela melhor solução da tarefa. Resultados similares foram encontrados em outros estudos, nos quais a capacidade da demonstração em informar "como fazer" foi superior à instrução verbal $^{36,49}$.

Em resumo, a demonstração tem mostrado melhores resultados com iniciantes que instrução verbal, principalmente na capacidade de informar "como fazer", e a combinação entre essas variáveis têm sido mais efetiva para a aprendizagem que de formas isoladas ${ }^{1,29,35,36,43}$. A efetividade da demonstração na aprendizagem motora está condicionada a alguns fatores, que estão relacionados às características do modelo, do observador e da tarefa, influenciando os quatro processos citados na teoria da Aprendizagem Social $^{34,35}$. Outro aspecto que pode influenciar nesses processos de representação simbólica são as operações de codificação simbólica. A importância dessas operações na aprendizagem por observação foi reforçada por meio de resultados que mostraram facilitação da aquisição de habilidades quando técnicas de codificação e ensaio mental foram empregadas ${ }^{28}$. Assim, a demonstração associada às técnicas de ensaio mental, pode ser uma importante estratégia para melhorar as operações de codificação simbólica, resultando em aprendizagem superior.

\section{Prática Mental}

Um fator determinante para a aquisição de habilidades motoras é a prática, que pode ocorrer de três formas: prática física, prática mental, e a combinação de prática física e mental ${ }^{10}$. A prática mental é definida como a recapitulação cognitiva de uma habilidade física na ausência de movimentos físicos explícitos $^{32}$.
Existem três formas de praticar uma habilidade mentalmente ${ }^{45}$ : a) auto-verbalização, que é a repetição mental e descrição verbal do movimento; b] auto-observação, que é a observação mental do próprio movimento, na qual o indivíduo se observa mentalmente praticando o movimento. Nesse caso, ele é o "expectador" da sua própria execução; e c) ideomotor, que é a imaginação e sensação cinestésica do próprio movimento, na qual o indivíduo executa mentalmente o movimento. Nesse caso, ele passa a ser o "ator" do movimento.

Tipicamente os estudos em aprendizagem motora comparam a prática mental com prática física e a combinação de prática física e mental. De forma geral, a prática mental não apresenta melhores efeitos que a prática física. Entretanto, a prática mental é melhor que a ausência de prática ${ }^{18,19,24,27,30}$.

Algumas teorias buscam fundamentar esses estudos, tentando esclarecer a estrutura e os mecanismos da prática mental, bem como sua relação com a melhora no desempenho e a que parece apresentar mais consenso na literatura é a Teoria da Aprendizagem Simbólica $^{48,51}$. De acordo com essa teoria, o processo de imaginação pode funcionar como um sistema codificador para ajudar as pessoas a entender e adquirir melhores padrões de movimentos. A teoria preconiza que a aprendizagem através da prática mental, ocorre basicamente pela repetição dos elementos simbólicos (cognitivos) da tarefa motora ${ }^{45,51}$.

Sendo assim, a teoria da aprendizagem simbólica tem forte relação com a aprendizagem observacional por modelação, uma vez que, a aprendizagem simbólica utiliza da observação de modelos (internos ou externos] como uma forma de 
tornar a prática mais eficiente. Ao adotar essa teoria, é possivel fazer uma inferência da relação entre demonstração e prática mental, já que ambas auxiliam na aprendizagem dos componentes simbólicos do movimento, sendo uma forma de tornar a prática mais eficiente.

\section{O Papel da Memória e a Relação entre Demonstração e Prática Mental}

Ao adotar a visão do ser humano como um processador de informações, o armazenamento da informação na memória é um dos aspectos mais importantes na aprendizagem de movimentos ${ }^{46}$. Esse armazenamento implica em três processos subseqüentes: o primeiro se refere à entrada de informação e correspondente à codificação; o segundo está relacionado ao armazenamento propriamente dito e o terceiro se refere à recuperação da informação armazenada, evocando a informação retida ou reconhecendo um conjunto particular dentre as informações apresentadas ${ }^{40}$. Essas proposições que atribuem à memória um papel fundamental na aprendizagem motora vão ao encontro das noções gerais da Teoria da Aprendizagem Social, na qual a aprendizagem motora ocorre através de códigos simbólicos e das transformações das informacões em imagens na memória. Os indivíduos quando realizam uma habilidade, comparam esta performance com o modelo interno na memória, fazendo julgamentos e corrigindo os erros para a próxima realização da habilidade e, a partir daí, o novo modelo é codificado na memória ${ }^{11}$.

A prática mental também pode ser considerada como um método para melhorar as codificações na memória e, esta codificação, pode ocorrer de duas formas: verbal e visual ${ }^{28}$.
Para que uma informação fique armazenada na memória de curto prazo, ela precisa ser ensaiada verbalmente ou através de imagens, e isso pode ser feito através da prática mental $^{31}$. 0 ensaio mental das imagens pode adquirir a forma de movimentos reais e ajudará no armazenamento de informações na memória. Sendo assim, técnicas de imaginação podem representar um facilitador desse processo, favorecendo a aprendizagem através da observação. As estratégias de memorização através da verbalização e imaginação representam uma forma eficaz na representação mental, favorecendo assim a aprendizagem observacional $^{22}$. A eficiência de tais estratégias pôde ser verificada em estudos nos quais as estratégias de ensaio mental incorporadas à demonstração, possibilitaram meIhor aprendizagem ${ }^{25,26,36,38,53}$. A combinação entre modelação e prática mental mostrou melhores resultados que a demonstração isolada ${ }^{3}$. Em síntese, esses resultados sugerem que a associação da demonstração e prática mental favorece a aquisição de habilidades motoras, e ainda, uma possível combinação entre demonstração e prática mental pode proporcionar aprendizagem superior que essas formas isoladas. Além disso, as proposições de que as paralelas entre modelação e imagem mental não podem estar distantes, parecem ser reforçadas $^{35}$. Esses autores sugerem que os processos que governam a aprendizagem observacional e prática mental são similares. Na aprendizagem observacional o indivíduo tipicamente observa a execução de determinada habilidade, as informações retiradas são codificadas e armazenadas para posterior reprodução física. Na prática mental, o indivíduo imagina a execução de uma habilidade, codifica e armazena as informações para então reproduzir a ação, suportando as idéias de que os processos internos de codificação e armazenamento de informações através da observação de um modelo e prática mental sejam parecidos.

São poucos ainda os estudos que avançaram nessa direção, representando um vasto campo de investigação em aprendizagem motora. Mais estudos manipulando a demonstração e prática mental de forma isolada e em combinação são necessários, para conhecer melhor quais aspectos essas variáveis afetam no processo de aquisição de habilidades, e se realmente, podem resultar em aprendizagem superior quando combinadas.

\section{CONCLUSÕES}

De acordo com as proposições da literatura, os processos que governam os aspectos relacionados à aprendizagem motora, pela demonstração e prática mental são similares, sugerindo que essas variáveis podem atuar de forma complementar na aquisição de habilidades motoras. Os resultados de estudos que utilizaram demonstração e prática mental apresentam indícios nessa direção, na qual a interação entre essas variáveis propiciou aprendizagem superior. Poucos estudos investigaram essas variáveis em conjunto com o objetivo de observar seus efeitos na aprendizagem. Sendo assim, a combinação de demonstração e prática mental se torna um vasto campo de investigação a ser atacado por pesquisadores do movimento humano. Testar como a interação entre essas variáveis afeta a aprendizagem do padrão da habilidade motora, assim como, o efeito de 
diferentes números de demonstrações e prática mental na aprendizagem de habilidades motoras, são sugestões para futuros estudos.

\section{CORRESPONDÊNCIA}

Fabiano de Souza Fonseca

Rua: Lindolfo de Azevedo 1723

apto 203, bairro Jardim América

Belo Horizonte - MG - Brasil

CEP 30460-050

E-mail: fabianoef@gmail.com

Tel.: 55-31-3373 5796

55-31-8765 5796

\section{REFERÊNCIAS}

1. Adams D (2001). The relative effectiveness of three instructional strategies on the learning of an overarm throw for force. The Physical Ed 58: 67-77.

2. Adams JA (1986). Use of the model's knowledge of results to increase the observer's performance. J Hum Mov Stud 12: 89-98.

3. Atienza FL, Balaguer I, Garcia-Merita, ML (1998). Video modeling and imaging training on performance on tennis service of 9- to 12-year-old children. Percept Motor Skills 87: 519-529.

4. Al-abood SA, Davids K, Bennett SJ (2001). Specificity of task constraints and effects of visual demonstrations in directing learners search during skill acquisition. J Motor Behav 33: 295-305.

5. Austin S, Miller L (1992). An empirical study of the sybervision golf videotape. Percept Motor Skills 74: 875-881.

6. Bandura A (1969). Principles of behavior modification. New York,
Holt, Rinehart \& Winston, Self-efficacy: toward a unifying theory of behavioral change. Psychological Review 84: 1215-91, 1977 In: McCullagh P. Modeling: learning, development, and social psychological considerations. In: Singer RN, Murphy, M, Tenant LK (Eds). Handbook of research in sport psychology. New York, McMillan.

7. Bandura A, Jeffery RW (1973). Role of symbolic coding and rehearsal processes in observational learning. J Pers Soc Psychol 26: 122-130.

8. Bandura A, Jeffery R, Bachica DL (1974). Analysis of memory codes and cumulative rehearsal in observational learning. J Res Pers 7: 295-305.

9. Bandura A (1986). Social foundations of thought and action: a social cognitive theory. Englewood Cliffs, NJ, Prentice-Hall.

10. Barela JA, Isayama HF (1995). Efeitos do tipo de prática na aprendizagem do estilo borboleta na natação. Rev Mov 2: 38-45.

11. Carroll WR, Bandura A (1982). The role of visual monitoring in observational learning of action patterns: making the unobservable observable. J Motor Behav 14: 153-167.

12. Carroll WR, Bandura A (1985). Role of timing visual monitoring and motor rehearsal in observational learning of action patterns. J Motor Behav 17: 269-281.

13. Carroll WR, Bandura A (1987). Translating cognition into action: the role of visual guidance in observational learning. J Motor Behav 19: 385-398.

14. Carroll WR, Bandura A (1990). Representation guidance of action production in observational learning: a causal analysis. J Motor Behav 22: 85-97.
15. Darido SC (1989). A demonstração na aprendizagem motora. Rev Kinesis 5: 169-178.

16. Darido SC (1991a). Efeito da demonstração na aquisição de uma habilidade motora. Rev Fund Esp Tur 3: 33-37.

17. Darido SC (1991b). Efeitos de dois procedimentos de apresentação da informação na aprendizagem motora: demonstração e instrução verbal. Dissertação de Mestrado não publicada, São Paulo: Escola de Educação Física/USP.

18. Driskell JE, Copper C, Moran A (1994). Does mental practice enhance performance? J Appl Physiol 79: 481-492.

19. Feltz DL, Landers DM (1983). The effect of mental practice on motor skill learning and performance: a meta-analysis. J Sport Psychol 5: 25-57.

20. Gerst MS (1971). Symbolic coding processes in observational learning. J Pers Soc Psychol 19: 7-17. 21. Godinho M, Mendes R, Melo F, Barreiros J (2002). Instrução e demonstração. In: Godinho, M [Eds]. Controlo motor e aprendizagem: fundamentos e aplicacõos. Cruz Quebrada: FMH edições, 151-162. 22. González MZ (2003) Posibilidades de la demonstración em el aprendizaje motor. Lecturas: Educacion Física y deportes 62, año 9. 23. Gould DR, Roberts GC (1982). Modeling and motor Acquisition. Quest Champaign 33: 214-30.

24. Grouios G (1992). Mental practice: a review. J Sport Behav 15:42-59. 25. Hall EG, Erffmeyer ES (1983) The effect of visuo-motor behavior rehearsal with videotaped modeling on free throw accuracy of intercollegiate female basketball players. J Sport Psychol 5:343-346. 26. Hall C, Moore J, Annett J, Rodgers W (1997). Recalling demonstrated and guided movements 
using imaginary and verbal rehearsal strategies. Res $Q$ Exercise Sport 68: 136-144.

27. Hird JS, Landers DM, Thomas JR, Horan JJ (1991). Physical practice is superior to mental practice in enhancing cognitive and motor task performance. J Sport Exerc Psychol 8: 281-293.

28. Jeffery R (1976). The influence of simbolic and motor rehearsal in observacional learning. J Res Pers 10: 116-127.

29. Landin $D$ (1994). The role of verbal cues in skill learning. Quest 46: 299-313.

30. Lejeune M, Decker C, Sanchez X (1994). Mental rehearsal in table tennis performance. Percept Motor Skills 79: 627-41.

31. Machado AA (1997). Atividade mental e performance motora. In: Pellegrini, AM (Org). Coletânea de Estudos: Comportamento Motor I. São Paulo: Movimento: 159-172.

32. Magill RA (2000). Aprendizagem motora: conceitos e aplicações. 5 a edição. São Paulo, Edgard Blucher. 33. Magill R, Schoenfelder-Zohdi B (1996). A visual model and knowledge of performance as sources of information for learning a rhythmic gymnastics skill. Int $J$ Sport Psychol 27: 7-22.

34. McCullagh P (1987). Model similarity effects on motor performance. J Sport Psychol 9: 249-260.

35. McCullagh P, Weiss M, Ross D (1989). Modeling consideration in motor skill acquisition and performance: an integrated approach. In: Pandolf $\mathrm{K}$ [Eds]. Exercise and sport sciences review. Baltimore, Williams \& Wilkins: 475-513.

36. McCullagh P, Stiehl J, Weiss MR (1990). Developmental modeling effects on the quantitative and qualitative aspects of motor performance. Res Q Exercise Sport 61: 344-350.
37. McCullagh P, Weiss MR (2001). Modeling: Considerations for motor skill performance and psychological responses. In: Singer RN, Hausenblas HA, Janelle CM [Eds] Handbook of Sport Psychology. 205-238. New York: John Wiley \& Sons.

38. Meaney KS (1994). Developmental modeling effects on the acquisition, retention, and transfer of a novel motor task. Res $Q$ Exercise Sport 65: 31-39.

39. Meaney KS, Griffin K, Hart MA [2005). The effect of model similarity on girls' motor performance. J Teaching Phys Ed 24: 165-178. 40. Melo F, Godinho M, Mendes R, Barreiros J (2002). Memória. In: Godinho M (Eds). Controlo Motor e aprendizagem fundamentos $e$ aplicações. Cruz Quebrada: FMH edições, 151-162.

41. Mendes R (2004). Modelo ou modelos? 0 que mostrar na demonstração. In: Barreiros J, Godinho M, Melo F, Neto C (Eds.). Desenvolvimento e aprendizagem: perspectivas cruzadas. Cruz Quebrada: FMH Edições, 95-117.

42. Newell KM, Morris LR, Scully DM (1985). Augmented information and the acquisition of skill in physical activity. In: Terjung RL [Eds]. Exercise and sport sciences review. New York: Macmillan, 235-261.

43. Públio NS, Tanl G, Manoel EJ (1995). Efeitos da Demonstração e Instrução Verbal na Aprendizagem de Habilidades Motoras da Ginástica Olímpica. Rev Paul Educ Fís 9: 11-124.

44. Richardson JR, Lee TD (1999). The effects of proactive and retroactive demonstrations on learning signed letters. Acta Psychol 101: 79-90.

45. Samulski D (2002). Psicologia do Esporte. São Paulo: Manole.
46. Schmidt RA, Wrisberg CA (2001). Aprendizagem e performance motora. Uma abordagem da aprendizagem baseada no problema. 2.ed. Porto Alegre: Artmed.

47. Shea C, Wright D, Wulf G, Whitacre C (2000). Physical and observational practice afford unique learning opportunities. J Motor Behav 32: 27-36.

48. Suinn R (1993). Imagery. In: Singer RN, Murphey M, TennanT LK (1993). Handbook of research on sport psychology. New York. Macmillan Publishing Company: 492-510.

49. Tillaar R, Ettema G (2003). Influence of instruction on velocity and accuracy of over arm throwing. Percept Motor Skills 96: 423-434. 50. Tonello MGM, Pellegrini AM (1997). A informação na aprendizagem motora: o modelo em destaque. In: PELLEGRINI AM [org.]. Coletânea de estudos: Comportamento Motor I. São Paulo: Movimento, 109-127.

51. Weinberg RS, Gould D (2001). Fundamentos da Psicologia do Esporte e do Exercício. 2.ed. Porto Alegre: Artmed.

52. Weiss, M, McCullagh P, Smith AL, Berlant, AR (1998). Observational learning and the fearful child: influence of peer models on swimming skill performance and psychological responses. Res $Q$ Exercise Sport 68: 380-394.

53. Weiss MR, Klint KA (1987). "Show and tell" in the gymnasium: an investigation of developmental differences in modeling and verbal rehearsal of motor skills. Res $Q$ Exercise Sport 58: 234-421.

54. Wulf G, Horstmann G, Choi B (1995). Does mental practice work like physical practice without information feedback? Res $Q$ Exercise Sport 66: 262-267. 